\title{
Diagnosis of Growth Hormone Excess and Hyperprolactinemia
}

\author{
Stefan Riedl · Herwig Frisch \\ Pediatric Department, Medical University of Vienna, Vienna, Austria
}

\section{Pathophysiology of Acromegaly and Hyperprolactinemia}

Acromegaly is a relatively rare disease that is caused by long-standing growth hormone $(\mathrm{GH})$ hypersecretion. The estimated prevalence of the disease is 60 cases per million with 3-4 new cases per million per year [1]. In exceptional cases, the disease is diagnosed prior to epiphyseal fusion, leading to pituitary gigantism. After completion of growth, the clinical symptoms become more similar to those in acromegalic adults like coarse facial features, acral changes, hyperhydrosis, headaches and visceromegaly.

In most cases, acromegaly is caused by pituitary adenomas, but it has become evident that lesions of hypothalamic, pituitary, or extracranial origin may be responsible for GH excess. Hypersecretion of GH-releasing hormone (GHRH) may be the result of a hypothalamic tumor, for example, hamartoma or ganglioneuroma, which either directly secretes GHRH or, alternatively, may stimulate hypothalamic GHRH production or impair the secretion of somatostatin. More frequently, GHRH secretion occurs from an ectopic GHRH-producing tumor like carcinoid tumors, pancreatic cell tumors, small cell lung cancer, and adrenal tumors [2]. GHRH may lead to GH hypersecretion and eventually pituitary acromegaly. GHRH is a potent mitogenic factor, and transgenic mice expressing the GHRH gene may develop somatotropic adenomas, which shows that GHRH is a direct tropic stimulus for the somatotropic cell [3]. Similarly, in McCune-Albright syndrome, constitutive activation of the GHRH-cAMP signaling pathway caused by an activating GNAS mutation leads to GH hypersecretion and pituitary adenoma formation in up to one third of patients [4, 5]. GHRH may also stimulate prolactin (PRL) secretion in acromegalic subjects, and about one third of such patients have hyperprolactinemia [1].

Several aspects favor the 'pituitary' hypothesis underlying acromegaly. In $90 \%$ of acromegalics, a pituitary tumor can be found, and these tumors can be plurihormonal, 
which implies that the hypersecretory cells must originate from a common stem cell. Furthermore, in nearly all cases the somatotropic tissue surrounding the tumor is not hyperplastic. This indicates that the primary disorder arises from a pituitary disorder. In addition, the high cure rate after selective adenomectomy and the low recurrence rate militate against a primary hypothalamic defect. The pituitary tumors may be pure GH cell adenomas, or they may be composed of two different cell types and secrete GH and PRL. The adenoma may also derive from the common GH and PRL stem cell and express both hormones. Plurihormonal tumors may express GH in combination with various other hormones such as ACTH or, rarely, TSH [5].

Still little is known about the genetic causes of common pituitary tumors. Nevertheless, the molecular basis of pituitary tumorigenesis in familial acromegaly and sporadic pituitary tumors has been elucidated during the last decade [6]: The occurrence of isolated familial somatotropinomas (IFS) was reported in 20 families and usually affects younger patients [7]. In familial isolated pituitary adenoma (FIPA), $50 \%$ of the adenomas are prolactinomas, with $\mathrm{GH}$-secreting and mixed $\mathrm{GH}-$ and PRL-secreting adenomas accounting for the remainder [8]. Moreover, mammosomatotroph pituitary adenomas may occur in MEN-1 [9] and the Carney complex [10].

Hyperprolactinemia is more frequent in females and leads to hypogonadism by impairing pulsatile gonadotropin release causing delayed menarche or menstrual irregularities/amenorrhea in girls and pubertal arrest in boys. In postpubertal subjects, symptoms of estrogen/testosterone deficiency like decreased libido, apathy and decreased bone mineral density predominate. In both sexes, rarely in boys, galactorrhea may occur. However, prolactin does not play a direct role in gynecomastia since it does not act as a growth hormone for the breast.

Hyperprolactinemia may be caused by a prolactinoma, which is the most frequent hormone-secreting pituitary tumor. Concordantly, a total of $45 \%$ of pituitary adenomas found in autopsy material were positive for PRL immunostaining. The age distribution of patients with these tumors was equal throughout the age range of 1686 years [11]. Depending on the size of the tumor, symptoms of a pituitary mass like visual abnormalities - most frequently bitemporal hemianopsia, opththalmoplegia or headache may occur. Another possible pathomechanism of elevated PRL levels is pituitary stalk compression by a tumor, leading to a decrease of PRL inhibition by dopamine. Besides mass effects, symptoms from pituitary insufficiency by compression of normal pituitary tissue may prevail in this case. Moderately elevated PRL levels may also result from interfering macroprolactin which can be differentiated by using polyethylene glycol precipitation.

\section{Regulation of Growth Hormone Secretion}

The secretion of GH is under the hypothalamic control of GHRH, which stimulates the synthesis and secretion of both GH and somatostatin. The latter acts to suppress 
the secretion of GH. The episodic pattern of GH secretion is predominantly regulated by fluctuations in somatostatin secretion [12]. Episodic secretion is also preserved in the presence of tonically elevated levels of circulating GHRH that results from an ectopic tumor [13].

Most of the growth-promoting effects of GH are mediated by insulin-like growth factor 1 (IGF-1), which exerts a negative feedback action on GH secretion and suppresses the pituitary synthesis of GH messenger ribonucleic acid (mRNA) [14]. IGF-1 is a GH-dependent peptide that is secreted mainly by the liver, but in addition, synthesis of IGF-1 also occurs in many tissues in an autocrine/paracrine manner. IGF-1 is age- and sex-dependent, but shows virtually no diurnal variation because it circulates in combination with a binding protein (BP). More than 95\% of IGF-1 is bound to insulin-like growth factor-binding protein 3 (IGFBP-3), a 150-kDa complex which acts as a carrier for IGF and regulates its bioavailability. IGFBP-3 is predominantly regulated by GH and reflects GH secretory status [see chapter by Blum et al., this vol.].

\section{Regulation of Prolactin Secretion}

PRL is secreted in a pulsatile manner, and there is diurnal variation with an increase in pulse amplitude after the onset of sleep. PRL is predominantly under an inhibitory influence of hypothalamic dopamine, whereas thyrotropin-releasing hormone (TRH), among other substances, is the main PRL-releasing factor. This explains why a mass lesion in the hypothalamic or pituitary stalk area, resulting in a decrease of anterior pituitary hormones, causes the elevation in PRL concentrations and why hyperprolactinemia is found in primary hypothyroidism, respectively. Other factors (e.g. vasoactive intestinal polypeptide (VIP), serotonin, opioid peptides, or GHRH) can stimulate PRL release; increased levels are also found during pregnancy and in chronic renal failure [15].

\section{Tests of Growth Hormone Secretion}

\section{Basal Serum GH Levels}

Increased GH secretion is the most prominent biochemical finding in acromegaly (see diagnostic algorithm, 'Appendix B, Chart 6'). The predictive value of random GH samples in identifying acromegaly varies with the selected threshold levels; however, elevated basal GH levels may occur in physiological or pathological conditions like stress (caused by the insertion of the cannula), pubertal growth spurt, exercise, diabetes, malnutrition, or chronic renal failure. Moreover, the spontaneous pulsatility of GH has to be taken into account. In addition, basal levels may be low in about $5 \%$ of acromegalic subjects. Nevertheless, acromegaly can be excluded if 
the GH level is less than $0.04 \mu \mathrm{g} / \mathrm{l}$ using newer, highly sensitive immunoradiometric assays [5].

Test Procedure. Several random blood samples should be taken from an inserted cannula over an extended period in order to avoid influences of stress, meals, etc. GH concentrations are then determined by immunoassay [see chapter by Ranke, this vol.].

\section{Spontaneous GH Pulses}

GH secretion shows a pulsatile pattern, and the secretory pulses are consequent to hypothalamic secretory bursts. The evaluation of GH pulsatility may thus make it possible to obtain information about hypothalamic GHRH secretion. In prepubertal children, a high correlation exists between relative height and the amount of GH secreted during a 24-hour period. In acromegalic patients, integrated 24-hour GH concentrations are significantly increased, and the pattern of spontaneous GH secretion is typically altered: the extent of pulsatile excursions is blunted, GH concentrations do not fall below the limit of detection, and the circadian pattern is abnormal, suggesting a defect in the neuroendocrine mechanisms regulating spontaneous $\mathrm{GH}$ secretion [5].

Test Procedure. Blood samples are collected for $24 \mathrm{~h}$ (or a defined shorter period) from an intravenous (i.v.) cannula, which permits undisturbed sampling while the subject is asleep. GH samples are taken every $20 \mathrm{~min}$, either by a continuously working withdrawal pump or by discrete samples. The total blood volume taken has to be considered, especially in younger subjects. Various parameters such as mean GH levels, pulse frequency, pulse amplitude, or area under the curve, can be calculated [see chapter by Albertsson-Wikland and Rosberg, this vol.]. Since GH secretion is age-dependent, it is important to compare the results with age-related reference values [see chapter by Albertsson-Wikland and Rosberg, this vol.]. This is a tedious method, which, however, gives reliable information about GH secretory status.

\section{Urinary GH Excretion}

Evaluation of urinary GH excretion has been developed as a noninvasive method for screening of GH status [16] However, this method is rarely used any more since great variation of GH excretion on a day-to-day basis exists. Moreover, methodological difficulties add to the low sensitivity and specificity of this test.

\section{GH Stimulation Tests}

Insulin Tolerance Test, Arginine Tolerance Test, and GHRH Test

The $\mathrm{GH}$ response to the classic $\mathrm{GH}$ provocation tests such as the insulin tolerance test (ITT) and the arginine tolerance test (ATT) in acromegalic patients may vary and can comprise a normal increase, no response, or a paradoxical decrease [17]. The stimuli act via the hypothalamic axis, but the tests are of no diagnostic or prognostic value. 
Similarly, stimulation with GHRH or GH-releasing peptide does not add to diagnostic information [18].

Dopamine Stimulation Test

In contrast to normal subjects, $\mathrm{GH}$ levels decrease by more than $50 \%$ in the majority of acromegalic individuals after the administration of dopamine agonists. The reason for this paradoxical reaction is a possible alteration of the somatotropic dopamine receptors. The function of this test is rather to identify possible responders to chronic therapy with a dopamine agonist.

Stimulation with TRH and Luteinizing Hormone-Releasing Hormone In a large proportion of acromegalic subjects, nonspecific $\mathrm{GH}$ release can be provoked by hypothalamic releasing hormones such as TRH or, less frequently, luteinizing hormone-releasing hormone (LHRH) [17]. The abnormal GH response disappears after successful therapy. These tests are of no special value for the diagnosis of acromegaly.

\section{GH Suppression}

\section{Oral Glucose Tolerance Test}

The oral glucose tolerance test (OGTT) is the most important test for confirming the diagnosis of acromegaly $[5,19,20]$. After oral glucose administration, GH levels decrease to levels $<1 \mu \mathrm{g} / \mathrm{l}$ in normal individuals. In acromegalic patients, GH levels are either not suppressible, or they demonstrate a paradoxical increase. In more than $90 \%$ of acromegalic subjects, GH does not decrease to $<1 \mu \mathrm{g} / \mathrm{l}$. Thus, the OGTT is a good screening procedure for acromegaly, although its sensitivity is limited by the fact that $7-10 \%$ of patients may nonetheless demonstrate suppression of GH levels to $<1 \mu \mathrm{g} / \mathrm{l}$. Moreover, other pathologic conditions such as diabetes, chronic renal failure, or severe malnutrition may be associated with elevated basal GH concentrations and prevent suppression of GH levels after the OGTT, thus limiting the specificity of the test. In addition, in tall children without pituitary disease, nonsuppression of GH after OGTT was found in a considerable percentage [21].

Test Procedure. The patients should be in a fasting and resting state for $12 \mathrm{~h}$ prior to commencement of the test. An oral glucose solution $(1.75 \mathrm{~g} / \mathrm{kg}$ body weight) is ingested in the first $5 \mathrm{~min}$ of the test (ready-to-drink containers of glucose solution are commercially available). Blood samples for the determination of glucose and GH levels are collected at $-30,0,30,60,90,120$ and $180 \mathrm{~min}$. It is important to carry out the test procedure for the entire 180-min duration. The test results are evaluated by taking into account the fact that normally GH levels decrease to $<1 \mu \mathrm{g} / \mathrm{l}$ within 60 120 min after the glucose load. High GH levels which do not decrease or which even display paradoxical increase are characteristic of acromegaly. 


\section{IGF-1 and IGFBP-3}

IGF-1

Patients with untreated acromegaly consistently have significantly elevated IGF1 levels that show no overlap with normal individuals [22]. It has been shown that increased IGF-1 levels are able to predict abnormal GH secretion early in the course of acromegaly, when $\mathrm{GH}$ levels are relatively low or decrease to $5 \mu \mathrm{g} / \mathrm{l}$ or less after glucose administration. Moreover, IGF-1 concentrations were significantly correlated to various parameters of disease severity such as clinical signs or blood glucose concentrations during the OGTT [23]. Thus, IGF-1 concentrations offer a highly accurate reflection of daily 24 -hour $\mathrm{GH}$ secretion $[5,19]$. In acromegalic individuals, a linear relation exists between IGF-1 and GH levels $<20 \mu \mathrm{g} / \mathrm{l}$. With higher GH levels, IGF-1 concentrations reach a plateau. IGF-1 is thus a sensitive indicator of GH hypersecretion. Even mildly elevated GH concentrations (5-10 $\mu \mathrm{g} / \mathrm{l})$ are associated with IGF-1 elevation. A single measurement of IGF-1 provides reliable information.

Test procedure. In order to determine the serum level of IGF-1, a random blood sample is drawn. After separation of the hormone from its BP by chromatography or extraction, the IGF-1 concentration is determined by immunoassay [see chapter by Blum et al., this vol.]. The test results are then compared with age- and sex-related reference data.

\section{IGFBP-3}

Serum IGFBP-3 levels are clearly elevated in untreated patients with acromegaly, and there is no overlap with the normal range. IGFBP-3 correlates significantly with the results of spontaneous $\mathrm{GH}$ secretion. In a large sample of untreated acromegalics with confirmed adenomas, IGFBP-3 was elevated even in those cases with normal or only slightly increased IGF-1 concentrations or in patients who showed GH suppression after a glucose load [24]. However, there is consensus that estimation of IGFBP-3 usually offers no advantage to IGF-1 in the assessment of disease activity [19].

Test procedure. IGFBP-3 is determined by immunoassay from a random blood sample. It is important to compare the results with age-related reference values [see chapter by Blum et al., this vol.].

\section{Other Diagnostic Tests in Acromegaly}

PRL

Basal PRL levels are elevated in $20-60 \%$ of patients with acromegaly [25]. Immunohistochemical studies have shown that all tumors contain PRL- and GHreactive cells [26]. Elevated PRL may also occur as a consequence of a pituitary stalk lesion resulting in an insufficient dopamine supply. Furthermore, hyperprolactinemia may be the consequence of augmented hypothalamic GHRH secretion, as it is frequently found in patients with ectopic GHRH secretion. GHRH injection also resulted 
in an increase in PRL [13]. The response of PRL to the administration of TRH in acromegalic patients may be normal, decreased or exaggerated and is therefore of no diagnostic help. Although clinical signs of GH excess already exist before hyperprolactinemia occurs, galactorrhea may be an early symptom in acromegaly. Likewise, any patient with hyperprolactinemia should have an evaluation of GH status.

\section{TSH and Gonadotropins}

$10-15 \%$ of pituitary adenomas in acromegaly show a plurihormonal character, producing also glycoproteinic hormones apart from GH and PRL. Plurihormonal adenomas occur more frequently in childhood and adolescence, and most of them (80\%) are macroadenomas [27]. In this context, basal TSH may be increased, but the TSH response to TRH may be impaired by elevated GH levels. If a deficiency of pituitary hormones also develops, gonadotropins are most frequently affected.

\section{Tests of Prolactin Secretion}

In general, PRL circulates in human plasma in 3 major variants: monomeric PRL (monoPRL; $23 \mathrm{kDa}$ ), dimeric PRL (bigPRL; 48-56 kDa); and polymeric forms (macroPRL; 150-204 kDa). MonoPRL is biologically active and accounts for the majority of immunoreactive PRL. MacroPRL results from an antigen-antibody complex of monoPRL and IgG and accumulates in serum due to its longer renal clearance. MacroPRLemia has been identified in $15-46 \%$ of hyperprolactinemic samples [28, 29]. Controversy exists as to biological effects of macroPRL, however, it is recommended to consider its occurrence if PRL levels and the clinical condition do not match, in order to avoid unnecessary radiological investigations or inappropriate treatment with dopamine agonists. Gel filtration chromatography (GFC) is the gold standard method for quantification of monoPRL, bigPRL and macroPRL, but too labor-intensive and expensive for use in clinical practice. In contrast, polyethylene glycol precipitation (PEG), a nonquantitative method, is easy to perform. It is therefore commonly used as a screening test since results correlate well with GFC [28].

For correct interpretation of PRL levels, it is essential to rule out hypothyroidism, chronic renal failure, pregnancy, and medication with neuroleptics, antihypertensive drugs, or calcium channel blockers. In general, normal basal PRL levels range from 5 to 15-25 ng/ml (upper limit range; higher in females). After TRH stimulation, the mean PRL level increases to $22.3 \pm 0.5 \mathrm{ng} / \mathrm{ml}$ (mean \pm SE) [30]. Slightly elevated basal PRL levels (25-40 ng/ml) may be idiopathic or result from emotional stress. Patients with PRLsecreting macroadenomas consistently exhibit markedly elevated PRL levels (>250 ng/ $\mathrm{ml}$, often $>1,000 \mathrm{ng} / \mathrm{ml}$ ). Using immunoradiometric methods, excessive PRL levels may sometimes lead to a 'hook' effect by saturation of antibody capacities and loss of labeled antibody, causing falsely low PRL values [28]. This effect can be overcome by serial dilutions. PRL levels up to $200 \mathrm{ng} / \mathrm{ml}$ may also be seen in acromegaly, since pituitary tumors 


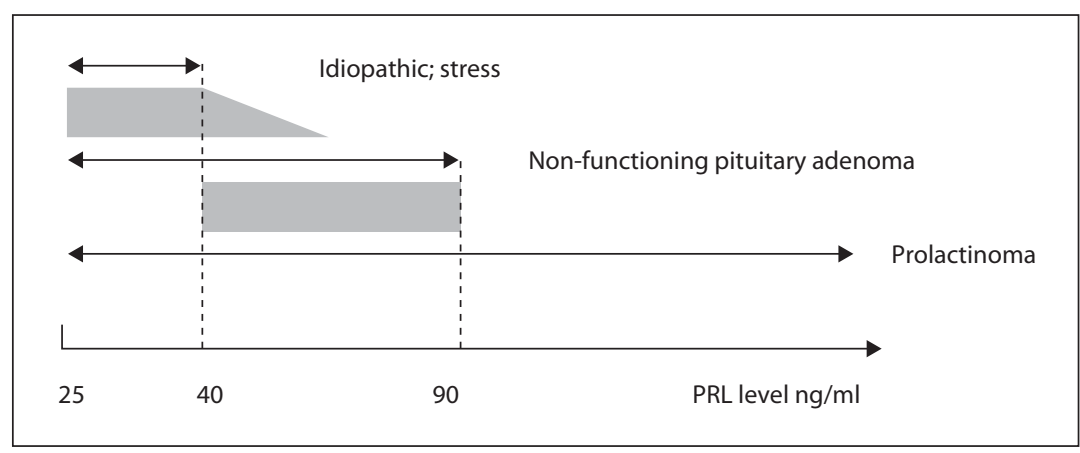

Fig. 1. Interpretation of elevated PRL levels. The overlap (shaded areas) between idiopathic/stressinduced hyperprolactinemia (PRL $25-40 \mathrm{ng} / \mathrm{ml}$ ) or non-functioning pituitary adenoma (PRL $<94 \mathrm{ng} /$ $\mathrm{ml}$ ) and PRL elevation caused by interfering macroPRL may be differentiated by PEG precipitation. Prolactinoma usually leads to much higher PRL levels. Data compiled from refrences 28 and 31.

may co-secrete GH and PRL. Pituitary tumors causing compression of the stalk show only moderate elevation of PRL $(<94 \mathrm{ng} / \mathrm{ml})$ [31]. Since there is a considerable overlap with 'false' hyperprolactinemia caused by macroPRL in that PRL range, it is essential to rule out the occurrence of macroPRL in such patients (fig. 1). The PRL response to TRH is characteristically blunted in patients with prolactinoma, but the response may be normal. Therefore, dynamic tests are not useful for the diagnosis [11].

Test Procedure. PRL tests are usually performed using two site immunoradiometric and chemiluminometric assays. Methods should be calibrated against the 3rd IRP WHO standard $84 / 500$ ( $1 \mu \mathrm{g} / \mathrm{l}$ is equivalent to $21.2 \mathrm{mIU} / \mathrm{l})$. To identify macroPRL, PEG dissolved in phosphate puffer saline (PBS) is added, before the sample is processed and the supernatant removed. Recovery is calculated by dividing the post-PEG PRL result by the PRL result obtained from the untreated sample. A PRL recovery of $>60 \%$ after PEG is found if monoPRL is present whereas a PRL recovery of $<40 \%$ is in line with macroprolactinemia [29]. PRL concentrations are usually reported in nanograms per milliliter.

For TRH test, see chapter by Krude and Grüters, this vol.

\section{Imaging Procedures}

Pituitary GH-producing tumors are the cause of more than $98 \%$ of acromegaly. Highresolution magnetic resonance imaging (MRI) of the pituitary-hypothalamic region is the method of choice for the detection of a possible tumor in this region. A resolution below $3 \mathrm{~mm}$ can be obtained when equipment with a high field strength (1.5-3 Tesla) is used. Spin-echo sequences, half-dose gadolinium injection, delayed sequence, and dynamic imaging add to diagnostic accuracy in small pituitary adenomas [32]. 
Evaluation of somatostatin receptors by scintigraphic imaging using radiolabeled somatostatin analogues may help to get information on the function of the tumor [33]. Dopamine antagonist binding to dopamine D2 receptors using PET or SPECT for visualization may discriminate prolactinomas and some GH secreting adenomas from nonfunctioning tumors; in addition, response to dopaminergic treatment may be predicted $[34,35]$.

\section{Special Aspects}

\section{Ectopic Origin of Acromegaly}

A rare cause of acromegaly is ectopic hormone secretion, which may be expected when elevated GH and GHRH levels are found in the absence of a primary lesion of the pituitary gland on MRI. Several types of extrapituitary tumors, such as carcinoid tumors, pancreatic cell tumors, small cell lung cancer, endometrial tumors, adrenal adenomas, and pheochromozytomas show positive GHRH immunostaining. Nevertheless, acromegaly in these patients is uncommon. If present, $\mathrm{GH}$ and IGF-1 concentrations are elevated, and GH fails to decrease after a glucose load. The response of GH to GHRH, TRH, or LHRH is of no help in distinguishing the central and the ectopic forms of acromegaly. The most useful discrimination parameter is the demonstration of elevated GHRH levels, which warrants further investigation for a peripheral tumor by abdominal and chest imaging. Furthermore, symptoms directly caused by the underlying tumor or unexpected clinical features like dyspnea, facial flushing, or peptic ulcers due to cosecretion of other substances may point at an ectopic origin of acromegaly [2].

\section{PRL Deficiency}

Occurrence of PRL deficiency (PRL $<5 \mathrm{ng} / \mathrm{ml}$ ) is generally rare. The incidence of acquired PRL deficiency rises with the number of other pituitary deficiencies and is usually seen in patients with craniopharyngioma, large nonfunctioning adenomas, pituitary apoplexy, and after pituitary surgery [36]. Congenital hypopituitarism including PRL deficiency may be caused by mutations in transcription factors and signaling molecules involved in pituitary development. If early developmental genes are affected, syndromic hypopituitarism may arise (HESX1: + septo-optic dysplasia; LHX3: + short cervical spine; limited neck rotation; sensorineural deafness; SOX3: + mental retardation; OTX2: + bilateral micro- or anophthalmia). The most prevalent monogenetic cause of non-syndromic combined pituitary hormone deficiencies is a PROP1 defect (GH, TSH, LH, FSH, PRL; evolving ACTH deficiency) and may cause pituitary enlargement on MRI. Mutations in POU1F1, which depends on temporal regression of $P R O P 1$ and is expressed throughout life, lead to GH, TSH and PRL deficiency, whereas corticotrophic and gonadotrophic functions are preserved [37]. 


\section{Conclusion}

Acromegaly is an extremely rare disorder before epiphyseal closure. The origin of $\mathrm{GH}$ hypersecretion may result from primary pituitary disease (approximately $98 \%$ of cases), hypothalamic GHRH secretion, or ectopic tumors. GH stimulates IGF-1 production, which in turn promotes the growth of multiple organs and tissues. A pragmatic approach to the diagnosis of acromegaly includes the detection of increased basal levels of GH, IGF-1 and IGFBP-3: this constitutes a simple screening method (see diagnostic algorithm 6). IGF-1 serum concentrations are always elevated in acromegaly. The OGTT represents the most important test in confirming the diagnosis of acromegaly. MRI is essential for detection of a pituitary tumor. Only the rare cases of ectopic hormone production will require further investigations.

Hyperprolactinemia may either result from a prolactinoma or from loss of dopamine-driven PRL suppression by pituitary stalk compression. PEG precipitation is used to overcome interference of macroPRL, causing elevated PRL levels without obvious clinical relevance.

\section{References}

1 Holdaway IM, Rajasoorya C: Epidemiology of acromegaly. Pituitary 1999;2:29.

2 Gola M, Doga M, Bonadonna S, Mazziotti G, Vescovi PP, Giustina A: Neuroendocrine tumors secreting growth hormone-releasing hormone: pathophysiological and clinical aspects. Pituitary 2006;9:221.

3 Asa SL, Kovacs K, Stefaneanu L, Horvath E, Billestrup N, Gonzalez-Manchon C, Vale W: Pituitary adenomas in mice transgenic for growth hormonereleasing hormone. Endocrinology 1992;131:2083.

- 4 Akintoye SO, Chebli C, Booher S, Feuillan P, 12 Kushner H, Leroith D, Cherman N, Bianco P, Wientroub S, Robey PG, Collins MT: Characterization of gsp-mediated growth hormone excess in the context of McCune-Albright syndrome. Clin Endocrinol Metab 2002;87:5104.

5 Melmed S: Acromegaly pathogenesis and treatment. J Clin Invest 2009;119:3189.

6 Horvath A, Stratakis CA: Clinical and molecular genetics of acromegaly: MEN1, Carney complex, McCune-Albright syndrome, familial acromegaly and genetic defects in sporadic tumors. Rev Endocr Metab Disord 2008;9:1.

7 Gadelha MR, Prezant TR, Une KN, Glick RP, Moskal SF 2nd, Vaisman M, Melmed S, Kineman RD, Frohman LA: Loss of heterozygosity on chromosome $11 \mathrm{q} 13$ in two families with acromegaly/gigantism is independent of mutations of the multiple endocrine neoplasia type I gene. J Clin Endocrinol Metab 1999;84:249.
8 Beckers A, Daly AF: The clinical, pathological, and genetic features of familial isolated pituitary adenomas. Eur J Endocrinol 2007;157:371.

$\$ 9$ Agarwal SK, Ozawa A, Mateo CM, Marx SJ: The MEN1 gene and pituitary tumours. Horm Res 2009; 71(suppl 2):131.

10 Boikos SA, Stratakis CA: Carney complex: the first 20 years. Curr Opin Oncol 2007;19:24.

11 Molitch ME: Prolactinoma; in Melmed S (ed): The Pituitary. Oxford, Blackwell Science, 1995, p443.

2 Tannenbaum GS, Ling N: The interrelationship of growth hormone (GH)-releasing factor and somatostatin in generation of the ultradian rhythm of GH secretion. Endocrinology 1984;115:1952.

13 Barkan AL, Shenker Y, Grekin RJ, Vale WW, Lloyd RV, Beals TF: Acromegaly due to ectopic growth hormone $(\mathrm{GH})$-releasing hormone $(\mathrm{GHRH})$ production: dynamic studies of GH and ectopic GHRH secretion. J Clin Endocrinol Metab 1986;63:1057.

14 Yamashita S, Weiss M, Melmed S: Insulin-like growth factor I regulates growth hormone secretion and messenger ribonucleic acid levels in human pituitary tumor cells. J Clin Endocrinol Metab 1986;63:730.

15 Freeman ME, Kanyicska B, Lerant A, Nagy G: Prolactin: structure, function, and regulation of secretion. Physiol Rev 2000;80:1523.

16 Girard J, Erb T, Pampalone A, Eberle AN, Baumann JB: Growth hormone in urine: development of an ultrasensitive assay applicable to plasma and urine. Horm Res 1987;28:71. 
17 Chang-DeMoranville BM, Jackson IM: Diagnosis and endocrine testing in acromegaly. Endocrinol Metab Clin North Am 1992;21:649.

18 Alster DK, Bowers CY, Jaffe CA, Ho PJ, Barkan AL: The growth hormone $(\mathrm{GH})$ response to $\mathrm{GH}$-releasing peptide (His-DTrp-Ala-Trp-DPhe-Lys-NH2), GHreleasing hormone, and thyrotropin-releasing hormone in acromegaly. J Clin Endocrinol Metab 1993;77:842.

19 Growth Hormone Research Society; Pituitary Society: Biochemical assessment and long-term monitoring in patients with acromegaly: statement from a joint consensus conference of the Growth Hormone Research Society and the Pituitary Society. J Clin Endocrinol Metab 2004;89:3099.

20 Carmichael JD, Bonert VS, Mirocha JM, Melmed S: The utility of oral glucose tolerance testing for diagnosis and assessment of treatment outcomes in 166 patients with acromegaly. J Clin Endocrinol Metab 2009;94:523.

-21 Holl RW, Bucher P, Sorgo W, Heinze E, Homoki J, Debatin KM: Suppression of growth hormone by oral glucose in the evaluation of tall stature. Horm Res 1999;51:20.

22 Brooke AM, Drake WM: Serum IGF-1 levels in the diagnosis and monitoring of acromegaly. Pituitary 2007;10:173.

23 Chen HS, Lin HD: Serum IGF-1 and IGFBP-3 levels for the assessment of disease activity of acromegaly. J Endocrinol Invest 1999;22:98.

-24 Grinspoon S, Clemmons D, Swearingen B, Klibanski A: Serum insulin-like growth factor-binding protein-3 levels in the diagnosis of acromegaly. J Clin Endocrinol Metab 1995;80:927.

25 de Pablo F, Eastman RC, Roth J, Gorden P: Plasma prolactin in acromegaly before and after treatment. J Clin Endocrinol Metab 1981;53:344.

26 Furuhata S, Kameya T, Otani M, Toya S: Prolactin presents in all pituitary tumors of acromegalic patients. Hum Pathol 1993;24:10.

-27 Scheithauer BW, Kovacs K, Randall RV, Horvath E, Laws ER Jr: Pathology of excessive production of growth hormone. Clin Endocrinol Metab 1986;15: 655.
28 Chahal J, Schlechte J: Hyperprolactinemia. Pituitary 2008;11:141.

29 McCudden C, Sharpless JL, Grenache DG: Comparison of multiple methods for identification of hyperprolactinemia in the presence of macroprolactin. Clin Chim Acta 2010;411:155.

30 Frisch H, Herkner K, Schober E, Stögmann W, Waldhauser F, Weissel M: Prolactin and thyrotrophin response to thyrotrophin-releasing hormone in growth hormone deficiency. Arch Dis Child 1982;57:769.

31 Karavitaki N, Thanabalasingham G, Shore HC, Trifanescu R, Ansorge O, Meston N, Turner HE, Wass JA: Do the limits of serum prolactin in disconnection hyperprolactinaemia need re-definition? A study of 226 patients with histologically verified non-functioning pituitary macroadenoma. Clin Endocrinol 2006;65:524.

32 Bonneville JF, Bonneville F, Cattin F: Magnetic resonance imaging of pituitary adenomas. Eur Radiol 2005; 15:543.

33 de Herder WW, Kwekkeboom DJ, Feelders RA, van Aken MO, Lamberts SW, van der Lely AJ, Krenning EP: Somatostatin receptor imaging for neuroendocrine tumors. Pituitary 2006;9:243.

34 Muhr C: Positron emission tomography in acromegaly and other pituitary adenoma patients. Neuroendocrinology 2006;83:205.

35 de Herder WW, Reijs AE, Feelders RA, van Aken MO, Krenning EP, van der Lely AJ, Kwekkeboom DJ: Diagnostic imaging of dopamine receptors in pituitaryadenomas. EurJEndocrinol 2007;156(suppl 1):S53.

-36 Toledano Y, Lubetsky A, Shimon I: Acquired prolactin deficiency in patients with disorders of the hypothalamic-pituitary axis. J Endocrinol Invest 2007;30:268.

37 Alatzoglou KS, Dattani MT: Genetic forms of hypopituitarism and their manifestation in the neonatal period. Early Hum Dev 2009;85:705.

Stefan Riedl, MD

St Anna Children's Hospital

Kinderspitalgasse 6

AT-1090 Vienna (Austria)

Tel. +43 140400 2800, Fax +43 140400 7280, E-Mail stefan.riedl@meduniwien.ac.at 